\title{
Acute descending necrotizing mediastinitis as a complication of the retropharyngeal abscess caused by anaerobes
}

\author{
Ilijaz Pilav${ }^{1}$, Orhan Čustović ${ }^{*}$, Arijana Horman-Leventa², Alma Alihodžić-Pašalić1, \\ Safet Mušanović ${ }^{1}$, Alen Pilav ${ }^{1}$, Kemal Grbić1, Kenan Kadić1, Meho Dapčević1, \\ Ademir Hadžismailovićc , Amela Katica-Mulalić
}

${ }^{1}$ Clinic of Thoracic Surgery, Clinical Center University of Sarajevo, Sarajevo, Bosnia and Herzegovina, ${ }^{2} \mathrm{Clinic}$ of Anaesthesiology and Intensive Care, Clinical Center University of Sarajevo, Sarajevo, Bosnia and Herzegovina

\begin{abstract}
Descending necrotizing mediastinitis (DNM) is a rare, life-threatening form of mediastinitis caused by odontogenic, pharyngeal, or cervical infections. The retropharyngeal space is the most common primary site of infection. Given the fulminant course and high mortality rate, early diagnosis and prompt treatment are important predictors of survival in patients with DNM. Appropriate empirical antibiotic treatment, prompt surgical intervention, and proper management of patients in the intensive care unit can be of vital importance. We present the case of a previously healthy 20-year-old male patient who was successfully cured and discharged from the Clinical Center University of Sarajevo after suffering from a severe form of mediastinitis as a complication of the retropharyngeal abscess caused by anaerobes.

Keywords: Descending necrotizing mediastinitis; mediastinum; retropharyngeal abscess; posterolateral thoracotomy
\end{abstract}

\section{INTRODUCTION}

Descending necrotizing mediastinitis (DNM) is a rare, acute infection of the mediastinal connective tissue, intrapleural space, and thoracic organs. It is characterized by a fulminant course and a mortality rate of up to $60 \%$ (1). It occurs most frequently as a complication of cervical necrotizing fasciitis caused by odontogenic infections, peritonsillar abscess,

*Corresponding author: Orhan Čustović, Clinical Center University of Sarajevo, Bolnička 25, Sarajevo, Bosnia and Herzegovina.

E-mail: orhan.custovic@gmail.com

Submitted: 09 August 2020/Accepted: 02 November 2020

DOI: https://doi.org/10.17532/jhsci.2020.1041 retro- and para-pharyngeal abscess, and epiglottitis. Communication of deep spaces of the head and neck through soft tissue structures, the force of gravity, and negative intrathoracic pressure during inspiration facilitate the spread of the purulent content from the neck to the mediastinum and pleura (2-4). Symptoms of mediastinitis can present within 12 hours up to 2 weeks after the onset of oropharyngeal infection, although most often within the first 48 hours. A delay in diagnosis and treatment contributes to high mortality rates. Early recognition of symptoms, followed by aggressive surgical treatment, prompt empirical antibiotic therapy, and adequate treatment in the intensive care unit (ICU), is essential to the survival of patients affected by extended 
DNM (5). We present the case of a previously healthy 20-year-old male patient with a severe case of DNM, who has been successfully treated thanks to a multidisciplinary team-based approach and discharged from the Clinical Center University of Sarajevo after 45 days of hospitalization.

\section{CASE REPORT}

A 20-year-old male patient was admitted through the Emergency Department (ED) to the ear, nose, and throat (ENT) clinic, the Clinical Center University of Sarajevo due to shortness of breath, neck pain, right shoulder pain, headache, and malaise. Several days before admission, the patient developed acute right otitis media, which was complicated by mastoiditis. His medical history was otherwise unremarkable. He had no history of tobacco, alcohol, or illicit drug use and was not taking any medications. Upon arrival to the ED, a chest X-ray (CXR) was performed (Figure 1), as well as an emergency contrast-enhanced computed tomography (CECT) scan of the head, neck, and chest (Figure 2). CXR revealed a widened superior mediastinum. CECT scan detected the presence of a hyperdense collection in the mastoid cells and tympanic cavity with signs of pneumocephalus. It also demonstrated multiple hypodense retropharyngeal collections with faint intermittent peripheral rim enhancement suggestive of a retropharyngeal abscess, with propagation

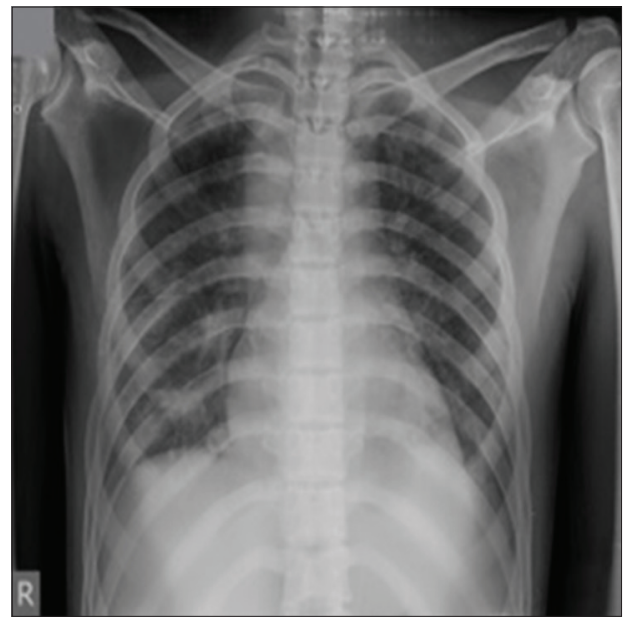

FIGURE 1. Anteroposterior radiograph of the chest upon arrival to the hospital emergency department. An ill-defined enlargement of the superior mediastinal shadow can be observed. across parapharyngeal spaces and caudally into the retroesophageal space. Furthermore, multiple reactive enhancing lymph nodes were present in the neck. Several rim-enhancing, multiloculated hypodense mediastinal collections suggestive of mediastinitis were also depicted, as well as bilateral lung abscesses and bilateral pleural effusions - moderate on the right and mild on the left side.

On the $2^{\text {nd }}$ day of admission to the ENT Clinic, the patient's condition deteriorated with signs of respiratory distress, hemodynamic instability, and septic shock. Right tube thoracostomy was an initial treatment of choice of progressive right-sided pleural effusion demonstrated on CXR. Considering the patient's condition, that is, markedly unstable patient with signs of progression from sepsis to septic shock, as well as the severity of the patient's respiratory failure, mediastinal drainage with right video-assisted thoracic surgery (VATS) was not performed at the time. The patient underwent surgical drainage of the right pleural cavity using a chest tube, which evacuated approximately $1300 \mathrm{cc}$ of pus. Moreover, physical examination revealed the presence of stridor and subcutaneous emphysema in the neck and upper chest. The incision in the left pectoral region was performed to evacuate the purulent content trapped beneath the skin, subcutaneous tissue, and fascia. During the following hours, the patient became unresponsive, tachycardic, and hypotensive (blood pressure was $90 / 60 \mathrm{mmHg}$ ). His heart rate was 85 beats per minute, he had no fever. To secure an adequate airway, endotracheal intubation was performed, and the patient was transferred to the ICU. Laboratory parameters upon admission were as follows: White blood cell count was $19.32 \times 10^{9} / \mathrm{L}$, red blood cell count was $2.53 \times 10^{12} / \mathrm{L}$, hemoglobin was $77.92 \mathrm{~g} / \mathrm{L}$, hematocrit was $23.12 \mathrm{~L} / \mathrm{L}$, platelet count was $113.60 \times 10^{9} / \mathrm{L}$, C-reactive protein was $271.5 \mathrm{mg} / \mathrm{L}$, and urea was $21.7 \mathrm{mmol} / \mathrm{L}$. The patient was connected to mechanical ventilation. He started receiving empirical antimicrobial therapy on the day of his ICU admission, that is, 2 days after the admission to the Clinical Center University of Sarajevo, and was given meropenem 2 g IV every 8 hours, metronidazole $500 \mathrm{mg}$ IV every 8 hours, and vancomycin $1 \mathrm{~g}$ IV every 12 hours. ENT surgeons performed an incision and drainage of the retropharyngeal abscess and abscess in the region of the neck 


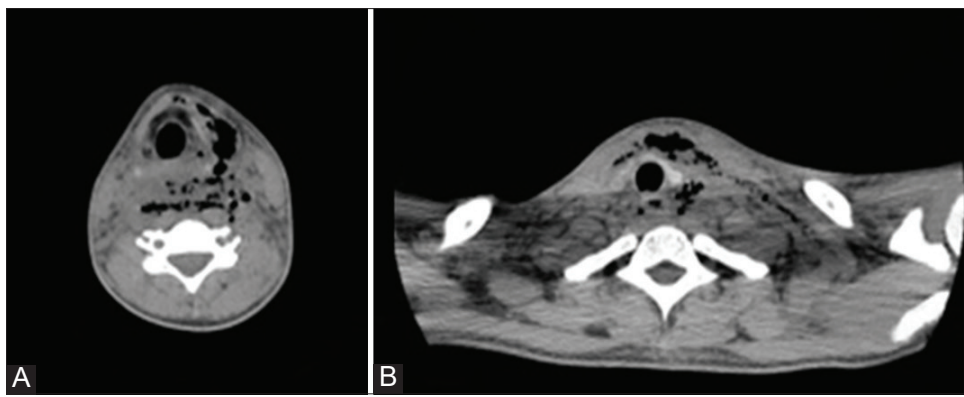

FIGURE 2. Computed tomography scans of the head and neck upon arrival to the hospital emergency department. (A) Abscesses in the para- and retro-pharyngeal spaces of the neck. (B) Multiple reactive enhancing lymph nodes in the neck, with multiple abscesses.

between the larynx and large blood vessels - the internal jugular vein and the common carotid artery. CT of the chest, performed on the $4^{\text {th }}$ day of admission to the ICU, demonstrated the progression of pleural effusions on both sides with multiple lung abscesses. VATS was considered but was not an option in this case, given the fact that the patient had already developed signs of septic shock and was hemodynamically compromised. Moreover, due to the severity of the patient's respiratory failure, he would not be able to tolerate single-lung ventilation. Wide access to the mediastinum and complete excision of the tissue necrosis was possible with an open thoracic approach. Emergency thoracotomy was indicated and the right posterolateral thoracotomy was performed, with decortication of the lung and incisions of the mediastinal pleura from the diaphragm to the pleural dome, along with incisions of the paratracheal, paracaval, and paraesophageal space. This was followed by a copious lavage of the thoracic cavity with sterile saline. Intraoperatively verified extensive pericardial effusion was managed with pericardial fenestration. After thoracotomy, three large (28 Fr) chest tubes were inserted into the right pleural cavity. An active pleural lavage was performed postoperatively for 8 days with continuous metronidazole solution through the cranial chest tube and pleural discharge evacuated by two remaining chest tubes under active suction. The goal of the continuous pleural lavage with metronidazole through chest tube was twofold: continuous irrigation of the pleural space and the lung, as well as the management of the infection caused by Gram-negative anaerobic bacteria. One chest tube was placed in the left pleural cavity and it evacuated approximately $1200 \mathrm{cc}$ of pus. CT of the chest, performed on the $2^{\text {nd }}$ post-operative day, demonstrated successful management of mediastinal infection through combined transthoracic mediastinal and cervical drainage (Figure 3). Transcervical drainage of the retropharyngeal abscess was performed on two more occasions, as well as the insertion of another chest tube in the left pleural cavity. Culture results revealed the presence of Prevotella melaninogenica and Bacteroides species; therefore, the antibiotics were tailored accordingly, that is, meropenem, clindamycin, and colistin were administered. The latter was associated due to nosocomial colonization. A follow-up CT scan of the chest detected recurrent pericardial effusion, which was monitored and managed conservatively. On the $20^{\text {th }}$ day of admission to the ICU, a general tonic-clonic (grand mal) seizure occurred; therefore, oxcarbazepine was administered. Due to prolonged intubation and unsuccessful separation of the patient from mechanical ventilation, a tracheotomy was performed with perioperative administration of tigecycline. Upon completion of previous antibiotic therapy, the infectologist indicated the continuation of antibiotic therapy with metronidazole and vancomycin.

The patient's condition gradually stabilized, and after a brief trial of spontaneous breathing, he was successfully liberated from mechanical ventilation. Chest tubes were sequentially removed. He was transferred to the clinic of thoracic surgery for additional treatment and underwent physical therapy to aid the recovery process. The CXR, taken before discharge from the hospital, demonstrated a significant radiographic improvement (Figure 4). After 45 days in the hospital, including 35 days in the ICU, the patient was discharged and transferred to a cantonal 


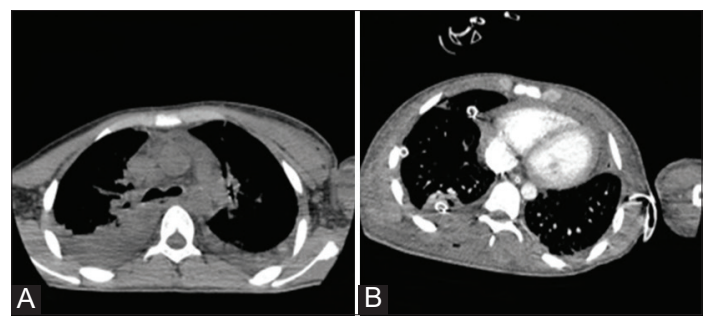

FIGURE 3. A comparison between computed tomography scans of the chest on day 1 of admission and after surgical treatment. (A) Bilateral pleural effusions can be observed. (B) Regression of pleural effusions indicates successful management of mediastinal infection through combined transthoracic and cervical drainage.

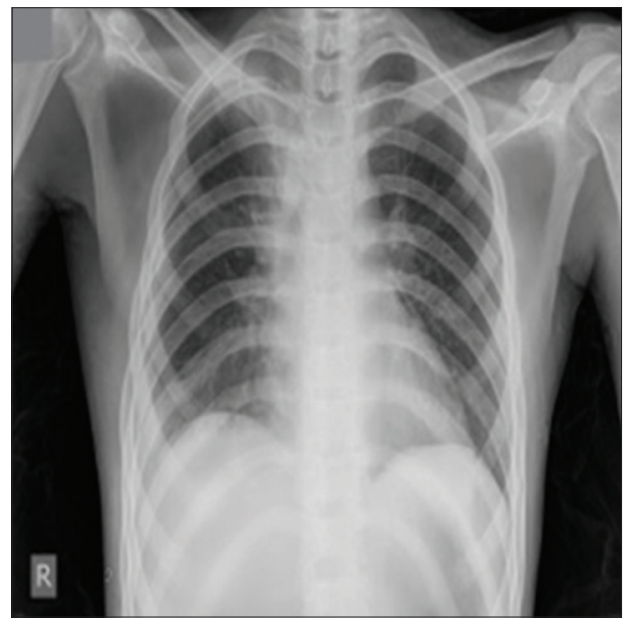

FIGURE 4. Chest radiograph taken before discharge from the clinic of thoracic surgery. The appearance of the lungs is significantly better than the day 1 admission appearance.

health care facility for further continuation of rehabilitation therapy.

\section{DISCUSSION}

The deep spaces of the head and neck, delimited by the deep cervical fascia, play a key role in the development of DNM. In about $70 \%$ of cases, DNM originates from the retropharyngeal space, with the infection spreading along the fascial planes into the mediastinum. This was also the case with our patient, in whom DNM developed as a complication of retropharyngeal abscess.

Risk factors for the development of oropharyngeal infection and DNM include older age, diabetes, alcohol and tobacco consumption, immunodeficiency disorders, and the use of immunosuppressive drugs $(6,7)$. The patient in our study is a 20-year-old man without pre-existing medical conditions and risk factors predisposing to the development of DNM. In one retrospective study, which included 45 patients with verified DNM, only 6 patients $(13.3 \%)$ did not have any of the aforementioned pre-existing comorbidities (5), confirming that DNM is an uncommon clinical entity in young, previously healthy people.

DNM is a polymicrobial infection - aerobes and anaerobes reflect the diversity of oropharyngeal microflora. In a retrospective study, which included 270 cases of oropharyngeal infection, the most common causative agent of DNM was $S$. pyogenes $(30.37 \%)$. Anaerobic bacteria were present in $16.3 \%$ of cases (8). Microbiological isolates in the case of our patient showed a predominance of anaerobic bacteria, with isolated $P$. melaninogenica and B. species. Estrera et al. defined the criteria used for the diagnosis of DNM: (a) Characteristic roentgenographic features of mediastinitis; (b) clinical evidence of severe oropharyngeal infection; (c) establishment of the relationship between DNM and the oropharyngeal process; and (d) documentation of necrotizing mediastinal infection at operation or post-mortem exam or both (2). Our patient met all of the criteria listed above.

A meta-analysis of Corsten et al. showed statistically significant improvement in the survival of patients who underwent neck and thoracic drainage (19\% mortality) versus transcervical drainage alone (41\% mortality, $p<0.05$ ) (9). A recent retrospective study of 45 patients diagnosed with DNM revealed that early diagnosis, combined with aggressive surgical and antibiotic treatment, may reduce the overall mortality rate to as low as $10-15 \%$ (5). With the increased popularity of minimally invasive surgery, the advantages of the VATS technique in the management of DNM were described by several authors. VATS approach allows excellent visualization of the entire thoracic cavity and it is reported to decrease morbidity when compared to thoracotomy and to result in better drainage of the mediastinum than transcervical drainage alone (10). During the course of his illness, our patient developed signs of septic shock and became hemodynamically as well as respiratory compromised. All these circumstances contraindicated the VATS procedure; 
therefore, an open thoracic approach was performed. Novel treatment options for patients with DNM also include immunotherapy, that is, combined treatment with IgM enriched immunoglobulin preparation and direct polymyxin B hemoperfusion, which was found to be successful in the treatment of a 38-year-old man with DNM (11), as well as hyperbaric oxygen therapy. The latter was first described by Kamiyoshihara et al. as an adjunctive treatment in resistant cases of DNM (12). In the case of our patient, posterolateral thoracotomy with wide incisions of the mediastinal pleura, debridement of devitalized tissue, and drainage of neck abscess, combined with a promptly given antimicrobial therapy were key components of successful treatment.

\section{CONCLUSION}

DNM is an uncommon, potentially fatal form of mediastinitis caused by odontogenic, pharyngeal, or cervical infections. A delay in diagnosis and treatment is associated with a fulminant course and high mortality rate. Studies have confirmed that aggressive surgical and antibiotic treatment in the early stages of infection significantly reduce complications and improve survival rates in patients with DNM. Recognizing and successfully treating DNM in young, previously healthy people remains one of the biggest challenges for clinical professionals.

\section{REFERENCES}

1. Gorlitzer M, Grabenwoeger M, Meinhart J, Swoboda H, Oczenski W, FiegIN, et al. Descending necrotizing mediastinitis treated with rapid sternotomy followed by vacuum-assisted therapy. Ann Thorac Surg 2007;83(2):393-6. https://doi.org/10.1016/j.athoracsur.2006.09.059.

2. Estrera AS, Landay MJ, Grisham JM, Sinn DP, Platt MR. Descending necrotizing mediastinitis. Surg Gynecol Obstet 1983;157(6):545-52.

3. Marty-Ane $\mathrm{CH}$, Alauzen $\mathrm{M}$, Alric $\mathrm{P}$, Serres-Cousine $\mathrm{O}$, Mary $\mathrm{H}$. Descending necrotizing mediastinitis. Advantage of mediastinal drainage with thoracotomy. J Thorac Cardiovasc Surg 1994;107(1):55-61. https://doi.org/10.1016/s0022-5223(94)70453-8.

4. Min HK, Choi YS, Shim YM, Sohn YI, Kim J. Descending necrotizing mediastinitis: A minimally invasive approach using video-assisted thoracoscopic surgery. Ann Thorac Surg 2004;77(1):306-10. https://doi.org/10.1016/s0003-4975(03)01333-x.

5. Ridder GJ, Maier W, Kinzer S, Teszler CB, Boedeker CC, Pfeiffer J. Descending necrotizing mediastinitis: Contemporary trends in etiology, diagnosis, management, and outcome. Ann Surg 2010;251(3):528-34. https://doi.org/10.1097/sla.0b013e3181c1b0d1.

6. Roccia F, Pecorari GC, Oliaro A, Passet E, Rossi P, Nadalin J, et al. Ten years of descending necrotizing mediastinitis: Management of 23 cases. J Oral Maxillofac Surg 2007;65(9):1716-24. https://doi.org/10.1016/j.joms.2006.10.060.

7. Gujrathi $A B$, Ambulgekar V, Kathait P. Deep neck space infection-a retrospective study of 270 cases at tertiary care center. World J Otorhinolaryngol Head Neck Surg 2016;2(4):208-13. https://doi.org/10.1016/j.wjorl.2016.11.003.

8. Mihos P, Potaris K, Gakidis I,Papadakis D, Rallis G. Management ofdescending necrotizing mediastinitis. J Oral Maxillofac Surg 2004;62(8):966-72. https://doi.org/10.1016/j.joms.2003.08.039.

9. Corsten MJ, Shamii FM, Odell PF, Frederico JA, Laframboise GG, Reid KR, et al. Optimal treatment of descending necrotising mediastinitis. Thorax 1997;52(8):702-8.

https://doi.org/10.1136/thx.52.8.702.

10. Guan X, Zhang WJ, Liang X, Liang X, Wang F, Guo X, et al. Optimal surgical options for descending necrotizing mediastinitis of the anterior mediastinum. Cell Biochem Biophys 2014;70(1):109-14. https://doi.org/10.1007/s12013-014-9865-z.

11. Pota V, Passavanti MB, Sansone P, Pace MC, Peluso F, Fiorelli A et al. Septic shock from descending necrotizing mediastinitis-combined treatment with IgM-enriched immunoglobulin preparation and direct polymyxin B hemoperfusion: A case report. J Med Case Rep 2018;12(1):55 https://doi.org/10.1186/s13256-018-1611-5.

12. Kamiyoshihara M, Hamada Y, Ishikawa S, lizuka T, Nakano M, Morishita Y. Hyperbaric oxygen as an adjunctive treatment for descending necrotizing mediastinitis: Report of a case. Kyobu Geka 2000;53 Suppl 8:715-7. 